\title{
Use of music streaming platforms for emotion regulation by international students
}

\author{
Greg Wadley, Amanda Krause \\ University of Melbourne \\ Parkville 3010, Australia \\ \{greg.wadley, amanda.krause\} \\ @unimelb.edu.au
}

\author{
Jiahui Liang, Zihe Wang \\ University of Melbourne \\ Parkville 3010, Australia \\ \{jiahuil7,zihew\} \\ @student.unimelb.edu.au
}

\author{
Tuck Wah Leong \\ University of Technology, Sydney \\ Ultimo 2007, Australia \\ tuckwah.leong@uts.edu.au
}

\begin{abstract}
Listening to music has always been an emotion-laden experience. Early research involving analog platforms showed that people use recorded music as a resource to manage their emotions, enhancing desired affective states and limiting unwanted states. More recently, technological advances such as streaming services have made an almost-unlimited selection of music ubiquitously available. This paper examines whether this intensified access to recorded music has afforded new forms of emotion regulation. We studied these practices in international university students, a cohort who face significant stresses and make significant use of digital technology. We found that students actively and routinely use music streaming services to manage their emotional responses to the challenges of studying abroad.
\end{abstract}

\section{CCS CONCEPTS}

- Human-centered computing Empirical studies in HCI

\section{KEYWORDS}

music listening; emotion regulation; music streaming platforms; international students;

\section{ACM Reference format: (LEAVE THIS TEXT AS IS FOR NOW, ACM} PROVIDES THE FINAL TEXT)

FirstName Surname, FirstName Surname and FirstName Surname. 2018. Insert Your Title Here: Insert Subtitle Here. In Proceedings of ACM Woodstock conference (WOODSTOCK'18). ACM, New York, NY, USA, 2 pages. https://doi.org/10.1145/1234567890

\section{Introduction}

Listening to music has always been an emotion-laden experience. Technological advances such as smartphones and digital streaming services have made an almost-unlimited selection of music ubiquitously available to many people [20].

*Article Title Footnote needs to be captured as Title Note

${ }^{\dagger}$ Author Footnote to be captured as Author Note

LEAVE THIS BOX AS IS FOR NOW, ACM PROVIDES THE FINAL TEXT:

Permission to make digital or hard copies of part or all of this work for personal or classroom use is granted without fee provided that copies are not made or distributed for profit or commercial advantage and that copies bear this notice and the full citation on the first page. Copyrights for third-party components of this work must be honored. For all other uses, contact the owner/author(s). WOODSTOCK'18, fune, 2018, El Paso, Texas USA
Prior research has shown that people use music as a resource to manage their emotions (e.g. [22]). More recently, research has begun to examine whether contemporary technology platforms affords new or more intensified forms of emotion regulation (e.g. [33]). At the same time, research has examined experiences arising from the use of music technologies in daily life (e.g. [25]).

The study reported here contributes to this body of work by examining how people use music streaming platforms to manage affect. We studied the practices of international university students, a cohort who face challenges that have begun to be examined in HCI research [27], [39]. We used a mainly qualitative approach that encouraged study participants to interpret their own experiences. We used this approach because:

1. Music platforms and practices are rapidly evolving, and exploratory studies are appropriate for discovering emerging phenomena ([23] p14);

2. Affective states such as emotions and moods are difficult to define and measure objectively [6];

3. The affective states that people seek to shape by listening to music are not necessarily the emotions typically examined in research [12].

We gathered data "in the wild" in order to capture people's experiences within the contexts of everyday life and everyday activities [24]. We present evidence that international students make use of music streaming services to manage their affective responses to the challenges of studying abroad.

\section{Related work}

\subsection{Music and mood regulation}

To function effectively in daily life, to behave appropriately in a social situation, or sometimes simply for hedonic reasons, people often seek to modify their affective states: a practice known as emotion regulation [13], [36]. Listening to music can powerfully impact a listener's emotions, and people often use music to shape affective states [16]. Music can distract a listener from a situation, relax them, reduce their stress, focus their thoughts, or help them to think about a situation in a new way [11], [33], [17]. Music can provide energy and motivation, and improve mood and performance during occupational [30] and household [2] tasks. People select music to align moods with activities [18], and choose energetic, rhythmic genres to 
upregulate mood and arousal [7]. People with depression [34] and neurosis [29] or who are recovering from addiction [9] use music to regulate affect. Music-based interventions have been designed to help adolescents regulate emotions [10].

\subsection{Digital music platforms}

Digital platforms give users greater control over music listening, and user-created playlists tend to reflect not genres but a user's contexts and reasons for listening [19], [21]. Personal, mobile listening allows users to construct auditory "bubbles" or life soundtracks [5]. Studies indicate that a third of music consumption via smartphones is undertaken for shaping affective states [33], and that office workers use music to control stress, distraction and personal space [14]. Streaming platforms make an almost-unlimited selection of music ubiquitously available [15]. It has been argued that Spotify, with playlists categorized by mood, provides personalized tools for enabling "pleasurable relief from ambient anxiety, boredom, and drudgery" [1]. Streaming services are used to manage employee [31] and customer [8] affect: evidence of the power of music to energize and refresh, to reduce stress, to manage concentration and distraction in contexts where work is done.

\subsection{Experiences of international students}

International students may experience problems due to cultural misunderstandings, discrimination, cultural isolation and loss of social support [38]. Most international students in western universities have a first language other than English, which can lead to language barriers and stress [32]. International students can face new life experiences, unfamiliar settings, financial stress, relationship-related issues and isolation [3] as well as pressure to succeed, migration issues and homesickness [26].The ability to self-regulate emotion is important to academic success, and US students use music to support self-regulation [35]. We hypothesized that international students studying in Australia might likewise turn to music to self-regulate their affective responses to student life, and that contemporary, especially international, streaming platforms might be valuable emotion regulation tools for these students.

\section{Method}

\subsection{Participants}

We recruited 10 international students, enrolled in a variety of degree courses at Australian universities. Half were female. They ranged in age from 23 to 30 . None were studying degrees that involved listening to music for study purposes. Participants did not interact with each other during the study

\subsection{Data collection}

Each participant underwent two phases of data collection: first using a diary to record their music listening experiences for 10 days; and second, taking part in individual interviews.

The researchers first discussed with participants ways in which they might keep a diary, including paper- and photo- based approaches. Participants emphasized that it was important that diary-keeping be easy. A solution based on social media was agreed upon: participants used chat channels to share their experiences with the researchers. This was acceptable to participants while allowing researchers to monitor participant engagement and ongoing data collection. For ten days participants recorded their use of music, along with their context, activities, challenges faced, and reasons for listening.

After the diary phase, each participant engaged in a semistructured interview, conducted either in person or via telephone. Some were conducted in the participant's native language, with transcripts translated into English for analysis. During interviews the participants' diary data was used to prompt recollection and reflection. Participants were asked about the type of music they like, in what situations they listen, and whether their patterns changed after moving overseas to study. They were asked whether music helped them deal with the affective states that arise in daily life, in particular those created by the challenges of international study.

\subsection{Data analysis}

Demographic data and the answers to some interview questions were characterized using descriptive statistics. Qualitative data from diaries and interviews were subjected to thematic analysis in order to identify, analyze and report patterns in the data [4].

\section{Results}

\subsection{Quantitative data}

While this is a primarily qualitative study and the sample is too small for hypothesis-testing, simple descriptive statistics are offered which characterize the participants and hint at trends that might be explored in future research.

\subsubsection{Frequency of listening to music}

Participants were asked whether they listened to music several times a day, almost every day, or not often. Male participants skewed towards "not often" while female participants said they listened "several times a day" or "almost every day".

\subsubsection{Genres of music listened to}

In order of frequency, participants reported listening to: hiphop, light (easy-listening) music, pop music, rock and roll, jazz, classical, and electronic music.

\subsubsection{Emotions associated with listening to music}

Participant diaries indicated a range of emotions associated with listening. Most often, participants chose to listen to music when they felt bored, fatigued, sad or relaxed. However, some participants did not clearly associate music listening with particular emotions, describing their affective state as "normal' or "ordinary". Male participants in particular tended to use more emotionally-neutral words in describing the circumstances in 
which they listened to music. (The extent to which this reflected perceived gender expectations was not determined.)

\subsubsection{Streaming services used}

Participants reported using a variety of streaming platforms. Some used more than one platform. In order of popularity these were: NetEase (4 participants), YouTube (3), Spotify (2), TenCent (2), iTunes (1), Xiami (1), QQ (1). NetEase Music is the most popular music app in mainland China, while participants mentioned that YouTube was attractive due to its enormous music resources.

\subsection{Qualitative data}

Analysis of diary and interview data yielded eight themes.

\subsubsection{Using music to boost energy and positive affect}

Fast-paced music, especially hip-hop, was the most common genre reported. Several participants reported using fast-paced music to boost their motivation, energy or arousal in order to get work done.

'I am writing an assignment and feel very tired. When I lack energy, I like to listen to hip hop music because hip hop music can bring me energy and keep my brain awake.' (Male, 28)

'I am dealing with ingredients I just bought from the supermarket. The same repeated work when dealing with ingredients makes me feel tired and sleepy. I need some fast-paced music to give me motivation.' (Male, 23)

"Cooking is [a] boring thing for me. Before I am cooking I need to play some music to make me energetic. When I am cooking with music, I feel that I could make a good meal." (Female, 26)

One participant used upbeat K-pop music to mitigate multiple affective states.

"Do you know about EXO, a pretty famous Korean boy band? I listen to their songs whenever I feel sad, boring, and even happy." (Female, 20)

\subsection{2 "Light" music as an aid to concentration}

After fast-paced music, the genre most mentioned was light or easy-listening music. This genre, which is pleasant and demands little of the listener, was often associated with the need to attain states conducive to work and study, such as focus or concentration.

'I am working in an internship company. I like to listen to light music when I work. Light music can make me concentrate better.' (Female, 24)

Light music helped some students achieve relaxation and concentration while also blocking distracting sounds.

'I am reading the materials in the library. I always listen to some slow-paced music while reading. Light music can let me concentrate and also feel relaxed. The library is sometimes not particularly quiet because some students will make noise, which will interfere with my attention. At this time, I will wear my headphones to listen to light music.' (Female, 28)

\subsubsection{Using music in response to boredom}

"Boredom" was the affective state most frequently cited as motivating music listening. Boredom was mentioned by every participant in regard to a variety of situations such as waiting, studying and working.

'I am waiting at the train station, there is nothing to do and I feel a little bit bored. I took out my mobile phone to listen to the song and chose a song from my favorite singer's new album. Listening to music when you are bored can really help you kill time.' (Female, 24)

'I came to the restaurant alone and just ordered the food. The food has not been done so I am waiting. Too boring, I started listening to music.' (Female, 23)

\subsubsection{Using music in response to stress}

Music was used to relieve stress related to busy schedules.

"I am listening to music and write my essay now. I need to find a suitable theme for my essay. It is not easy. So I am really upset now." (Female, 26)

"I was listening to music on the way to school. I didn't choose what to listen on purpose, just shuffled the playlist and listened to songs with strong rhythm. I was about to be late this morning but due to music, I feel less stressed out and anxious than I supposed to be, and walk faster than usual." (Male, 25)

However, when experiencing negative emotions, some participants preferred to use regulation strategies other than listening to music.

'I don't think music can greatly alleviate my negative emotions. When I have negative emotions, I often eat and drink with friends or play online games myself.' (Male, 28)

\subsubsection{Using music for peace of mind}

Some listened to music for quiet enjoyment and to attain a peaceful state of mind. (This may a form of stress reduction, as discussed in the previous section.)

"I listened to Korean songs through Spotify without reason. This seems like a form of quiet enjoyment, not having to think about anything." (Female, 27)

\subsubsection{Using music in response to homesickness}

Participants reported feeling homesick and lonely during the period they had been away from their home country.

'I called home today and talked to my parents about my life abroad. I miss the days when my parents and friends are with me.' (Female, 23)

To alleviate homesickness, some students listened to songs related to family or country.

'I just finished my work and walked home. I suddenly felt very homesick, especially missed my mother, so I listened to a song about maternal love.' (Female, 24)

4.2.7 Changes in music preferences while studying abroad 
About half the participants felt their music preferences had changed since studying abroad. For example, one female participant did not often listen to rap before going abroad but had come to like this genre, because listening to it gave her courage in her new environment and made her feel less lonely when traveling home late from work. On the other hand, the use of international streaming services allowed participants to maintain familiar listening habits.

\subsubsection{Use of streaming and recommender systems}

Participants almost universally used (and praised) music streaming platforms, along with their associated features such as playlists and recommendation systems.

"It's quite a long time that I haven't bought any physical album from offline shop that I am not willing to collect." (Female, 20)

"I like to use streaming services, mostly YouTube. I can listen to all kinds of music or watch their videos without limitations. Unlike albums, there are just a few songs that maybe not are my taste." (Male, 22)

Features such as playlists and recommender systems allowed participants to choose music that matched context and activity:

I can listen to a specific type of music when I do some stuff. When I walk to school, I like to listen [to] fast paced songs. But if I stay at home, I will enjoy light music because of assignments. One function resolves a lot of problems, which is cool." (Male, 25)

However, recommender systems were not always satisfactory:

"My music application Netease provided some strange songs to me, I did not listen to them because of weird names. Thus, I prefer Xiami app because it often gives me lots of songs related to my favor." (Female, 26)

\section{Discussion}

This study examined the music listening practices of international students studying in Australia, with a focus on the use of streaming services as emotion regulation tools. The challenges experienced by these students such as workload, pressure to succeed, unfamiliar culture, and distance from home create affective states such as stress, boredom, fatigue and loneliness, and sometimes joy. This study provides evidence that students use the vast musical resources of contemporary streaming services and the control afforded by playlists and personal devices in attempts to shape their affective states.

Both hedonic and instrumental emotion regulation ([36]) was evidenced in our study: for example, students listened to music to reduce unpleasant states such as sadness or boredom (hedonic motive), but also to boost energy and concentration in order to work more effectively (instrumental motive). Furthermore, it appears that music offers a way to regulate affect even when people can't define their emotional states.

Our data confirm earlier findings that people use music to reduce unwanted emotional states and to attain preferred states (e.g. [22][12][19]). The novel contribution of this study is a focus on emotion regulation involving (a) digital streaming services and (b) the challenges and practices of international students. Thus, this work contributes to knowledge about digital emotion regulation, but also to knowledge about students' use of emerging technologies to respond to the pressures of study, especially study undertaken abroad. It also suggests avenues for design work, since the features offered by streaming platforms did not always suit the users' needs: this could be relevant to vendors of streaming services as well as to designers of musicbased interventions.

We frequently observed the use of music as a "boredom buster" or time filler. Boredom has been called "a desire for desires" [37]. It is something that people try to 'escape', and music is a resource recruited in people's escapades. Yet boredom does not necessarily indicate an absence of emotion, as it can foster rumination, which is sometimes masked with media use [12]. Boredom is also associated with a decrease in energy, suggesting that arousal through energetic, fast-paced music can be an antidote to boredom.

Music as a cure for homesickness has been mentioned in historical accounts of long-distance travel [28]. It will be interesting in future research to investigate how internationallybased music streaming services, accessed through personal, devices, are impacting the experience of travel, study and work abroad: To what extend does ubiquitous access to a vast library of multicultural music, possibly bolstered by mediated news and social connection, empower travelers to battle homesickness?

\subsection{Limitations and future research}

This exploratory study used qualitative methods to gather data from a relatively small number of students at Australian universities. Future research could build on these findings by recruiting larger samples and comparing the practices reported here with the practices of students from other backgrounds and studying in other countries. Future research could examine in more detail whether different platforms and streaming services offer different affordances for emotion regulation, and how streaming services are adopted and translated internationally by students and other travelers.

\section{Conclusion}

This study examined the use of music streaming services for emotion regulation by international university students, a cohort who face significant stresses in daily life, and make significant use of digital technology. We found that students make routine use of music streaming services to manage their emotional responses to the challenges of studying abroad. For this group, practices that involve using music as a tool for emotion regulation appear to be evolving with emerging technologies and platforms. It appears that the power of music to shape affective states is retained and indeed refined by digital streaming services.

\section{ACKNOWLEDGMENTS}

We thank the participants who gave up their time to take part in this study, as well as colleagues in the Digital Emotion Regulation group at Melbourne for insightful discussions. 


\section{REFERENCES}

[1] Anderson, P.A. 2015. Neo-muzak and the business of mood. Critical Inquiry. 41, 4 (2015), 811-840. DOI:https://doi.org/10.1086/681787.

[2] Bauer, C. and Kratschmar, A. 2015. Designing a music-controlled running application: A sports science and psychological perspective. Conference on Human Factors in Computing Systems - Proceedings. 18, 2015 (2015), 1379-1384. DOI:https://doi.org/10.1145/2702613.2732736.

[3] Bradley, G. 2000. Responding effectively to the mental health needs of international students. Higher Education. 39, 4 (2000), 417-433.

[4] Braun, V. and Clarke, V. 2006. Using thematic analysis in psychology. Qualitative research in psychology. 3, 2 (2006), 77-101.

[5] Bull, M. 2015. Sound moves: iPod culture and urban experience. Routledge.

[6] Calvo, R.A. and D'Mello, S. 2010. Affect detection: An interdisciplinary review of models, methods, and their applications. IEEE Transactions on Affective Computing. 1, 1 (2010), 18-37. DOI:https://doi.org/10.1109/TAFFC.2010.1.

[7] Cook, T. et al. 2019. Music as an emotion regulation strategy: An examination of genres of music and their roles in emotion regulation. Psychology of Music. 47, 1 (2019), 144-154. DOI:https://doi.org/10.1177/0305735617734627.

[8] DeNora, T. 2000. Music in everyday life. Cambridge University Press.

[9] Dingle, G.A. et al. 2015. The influence of music on emotions and cravings in clients in addiction treatment: A study of two clinical samples. Arts in Psychotherapy. 45, (2015), 18-25 DOI:https://doi.org/10.1016/j.aip.2015.05.005.

[10] Dingle, G.A. and Fay, C. 2017. Tuned In: The effectiveness for young adults of a group emotion regulation program using music listening. $\begin{array}{llllll}\text { Psychology of } \quad \text { Music. } & 45, \quad 4 & \text { (2017), } & \text { 513-529. }\end{array}$ DOI:https://doi.org/10.1177/0305735616668586

[11] Van Goethem, A. and Sloboda, J. 2011. Article The functions of music for affect regulation Annelies van Goethem. Musicae Scientiae. 15, 2 (2011), 208-228. DOI:https://doi.org/10.1177/1029864911401174.

[12] Greenwood, D.N. and Long, C.R. 2009. Mood specific media use and emotion regulation: Patterns and individual differences. Personality and Individual Differences. 46, 5-6 (2009), 616-621. DOI:https://doi.org/10.1016/j.paid.2009.01.002.

[13] Gross, J.J. 1998. The emerging field of emotion regulation: An integrative review. Review of general psychology. 2, 3 (1998), 271-299.

[14] Haake, A.B. 2011. Individual music listening in workplace settings: An exploratory survey of offices in the UK. Musicae Scientiae. 15, 1 (2011), 107-129. DOI:https://doi.org/10.1177/1029864911398065.

[15] Hosey, C. et al. 2019. Just Give Me What I Want: How People Use and Evaluate Music Search. Proceedings of the 2019 CHI Conference on Human Factors in Computing Systems (2019), 299.

[16] Juslin, P.N. and Laukka, P. 2004. Expression, Perception, and Induction of Musical Emotions: A Review and a Questionnaire Study of Everyday Listening. Journal of New Music Research. 33, 3 (2004), 217-238. DOI:https://doi.org/10.1080/0929821042000317813.

[17] Knight, W.E.J. and Rickard, N.S. 2001. Relaxing music prevents stressinduced increases in subjective anxiety, systolic blood pressure, and heart rate in healthy males and females. Journal of music therapy. 38, 4 (2001), 254-272.

[18] Knobloch, S. 2003. Mood adjustment via mass communication. Fournal of $\begin{array}{llll}\text { Communication. } & 53, & 2 & \text { (2003), }\end{array}$ DOI:https://doi.org/10.1093/joc/53.2.233

[19] Krause, A. et al. 2014. Music selection behaviors in everyday listening. fournal of Broadcasting \& Electronic Media. 58, 2 (2014), 306-323.

[20] Krause, A.E. and Hargreaves, D.J. 2013. MyTunes: Digital music library users and their self-images. Psychology of Music. 41, 5 (2013), 531-544. DOI:https://doi.org/10.1177/0305735612440612.

[21] Krause, A.E. and North, A.C. 2014. Contextualized music listening: playlists and the Mehrabian and Russell model. Psychology of Well-Being. 4, 1 (2014). DOI:https://doi.org/10.1186/s13612-014-0022-7.

[22] Larson, R. 1995. Secrets in the Bedroom: Adolescents 'Private Use of Media. Fournal of Youth and Adolescence. 24, 5 (1995), 535-550.

[23] Lazar, J. et al. 2017. Research methods in human-computer interaction. Morgan Kaufmann.

[24] Leong, T.W. et al. 2010. Understanding experience using dialogical methods: The case of serendipity. ACM International Conference $\begin{array}{llr}\text { Proceeding } & \text { Series. } & \text { (2010), }\end{array}$ DOI:https://doi.org/10.1145/1952222.1952278.

[25] Leong, T.W. and Wright, P. 2013. Revisiting social practices surrounding music. Conference on Human Factors in Computing Systems - Proceedings. (2013), 951-960. DOI:https://doi.org/10.1145/2470654.2466122.

[26] Li, C.-S. and Lin, Y.-F. 2014. Understanding Asian International College Students' Values and Beliefs, their Acculturative Stress and Coping Strategies. Journal of Education and Human Development. 3, 4 (2014), 5964. DOI:https://doi.org/10.15640/jehd.v3n4a5.

[27] Malone, L. 2019. Finding Themselves between Home and Host Cultures. Companion Proceedings of The 2019 World Wide Web Conference (2019), $42-45$.

[28] Matt, S.J. 2011. Homesickness: an American history. Oxford University Press.

[29] Miranda, D. and Blais-Rochette, C. 2018. Neuroticism and emotion regulation through music listening: A meta-analysis. Musicae Scientiae. (2018), 1029864918806341.

[30] Oldham, G.R. et al. 1995. Listen while you work? Quasi-experimental relations between personal-stereo headset use and employee work responses. Fournal of Applied Psychology. 80, 5 (1995), 547.

[31] Plourde, L 2017. Sonic air-conditioning: Muzak as affect management for office workers in Japan. Senses and Society. 12, 1 (2017), 18-34. DOI:https://doi.org/10.1080/17458927.2017.1268812.

[32] Poyrazli, S. et al. 2004. Social support and demographic correlates of acculturative stress in international students. Fournal of College Counseling. 7, 1 (2004), 73-82.

[33] Randall, W.M. and Rickard, N.S. 2017. Reasons for personal music listening: A mobile experience sampling study of emotional outcomes. Psychology of Music. 45, 4 (2017), 479-495. DOI:https://doi.org/10.1177/0305735616666939.

[34] Sakka, L.S. and Juslin, P.N. 2018. Emotion regulation with music in depressed and non-depressed individuals. Music \& Science. 1, (2018), 205920431875502. DOI:https://doi.org/10.1177/2059204318755023.

[35] Stapley, J.C. 2014. Music and emotion regulation among emerging adults in college. Adolescence in the 21st century: Constants and challenges. (2014), 225-238.

[36] Tamir, M. 2016. Why Do People Regulate Their Emotions? A Taxonomy of Motives in Emotion Regulation. Personality and Social Psychology Review. 20, 3 (2016), 199-222. DOI:https://doi.org/10.1177/1088868315586325.

[37] Tolstoy, L. 1878. Anna Karenina (Oprah\# 5):(Penguin Classics Deluxe Edition). Penguin.

[38] Yakunina, E.S. et al. 2010. Group counseling with international students: Practical, ethical, and cultural considerations. Fournal of College Student Psychotherapy. 25, 1 (2010), 67-78.

[39] Yuan, C.W. et al. 2014. International students' use of facebook vs. a home country site. CHI'14 Extended Abstracts on Human Factors in Computing Systems (2014), 2101-2106. 